\title{
YouTube as a source of information on gout: a quality analysis
}

\author{
Mustafa Erkut Onder ${ }^{1}\left[\right.$ O Orhan Zengin ${ }^{2}$ (D)
}

Received: 15 January 2021 / Accepted: 13 February 2021 / Published online: 1 March 2021

(c) The Author(s), under exclusive licence to Springer-Verlag GmbH, DE part of Springer Nature 2021

\begin{abstract}
YouTube is a popular video-sharing platform commonly visited by patients and healthcare professionals for medical information. Gout is the most frequent cause of inflammatory arthritis in adults. However, the accuracy and quality of gout-related information on YouTube are not fully known. Thus, the aim of this study was to assess the reliability and quality of YouTube videos pertaining to gout. A YouTube search was conducted using the keywords "gout", "gout arthritis", "gout treatment", and "gout diet". Of the 240 videos screened, 114 that met the inclusion criteria were included in the study. The number of days since upload, number of views, likes, dislikes, comments and duration of the videos were recorded. A Modified DISCERN tool and the Global Quality Scale (GQS) were used to evaluate the reliability and quality of the videos. Of the 114 analyzed videos, $87.72 \%$ were deemed as useful while $12.28 \%$ contained misleading information with higher viewership. The most common topic was "gout diet". The majority of the videos demonstrated high quality (57.89\%), followed by intermediate quality $(28.95 \%)$ while the percentage of low-quality videos was $13.16 \%$. Videos posted by academic institutions/professional organizations and physicians had higher modified DISCERN and GQS scores indicating higher reliability and quality. This study demonstrated that the majority of YouTube videos on gout provide useful information. However, physicians should be aware of the limited nature of YouTube and correct any misinformation during face-to-face meetings. YouTube should consider avoiding misleading videos using validity scales such as modified DISCERN and GQS.
\end{abstract}

Keywords YouTube $\cdot$ Gout $\cdot$ Internet $\cdot$ Social media $\cdot$ Quality

\section{Introduction}

In recent years, the internet has become a popular source of health information [1]. Previous studies demonstrated that $80 \%$ of internet users access health information online [2], with YouTube being the most common video-sharing platform and the second most popular website worldwide after Google Search [3]. More than $500 \mathrm{~h}$ of content are uploaded to YouTube every minute, and people watch over a billion hours of video and generate billions of views every day [4]. As a video-sharing platform, YouTube has gone beyond normal text-based information and become a potential source

Mustafa Erkut Onder

erkutonder@hotmail.com

Orhan Zengin

drorhanzengin@gmail.com

1 Department of Rheumatology, Aksaray University Training and Research Hospital, 68200 Aksaray, Turkey

2 Department of Rheumatology, Gaziantep Dr. Ersin Arslan Training and Research Hospital, 27500 Gaziantep, Turkey for spreading information in the field of health [5]. On the other hand, $86 \%$ of health seekers are concerned about the reliability of information available on the internet, and $44 \%$ believe only part of this information to be accurate [6]. Since YouTube has no procedure for filtering videos before posting, viewers can also be exposed to misinformation [7]. Many recent YouTube studies have drawn attention to the importance of tackling misleading information on rheumatic diseases $[5,8,9]$.

Gout, described as "the king of diseases and the disease of kings", is one of the oldest diseases which was first recognized by the Egyptians in 2640 BCE [10]. The disease is the most frequent cause of inflammatory arthritis that affects approximately $4 \%$ of adults in the USA [11, 12]. It occurs due to the accumulation of monosodium urate crystals in joints or soft tissues [13]. Clinical disease arises as a consequence of the inflammatory reaction of tissues to these deposited crystals [11]. Gout is the only chronic arthritis potentially curable with the long-term use of appropriate medications [14]. The European League Against Rheumatism (EULAR) and American College of Rheumatology 
(ACR) guidelines for the management of gout recommend lifestyle modifications such as diet, weight loss, and exercises alongside pharmacologic treatment $[15,16]$. However, this condition is frequently poorly managed in the majority of patients [14]. Gout is increasingly being considered as a serious disease that causes functional disability, work productivity impairment, and economic loss [17].

Decisions made by three quarters of patients on how to treat their condition are reported to be affected by information provided by online searches [2]. YouTube is one of the most prevalent sources of online health information [18]. However, there are concerns about the reliability and quality of online health information [7]. To our knowledge, there is no study that evaluated the content, reliability, and quality of gout-related videos posted on YouTube. Hence, this study was performed to investigate the content, reliability and quality of health information pertaining to gout on YouTube.

\section{Materials and methods}

YouTube (http://www.youtube.com) was searched using the keywords "gout", "gout arthritis", "gout treatment", and "gout diet" on December 1, 2020. As most viewers do, the default YouTube "relevance" filter applied for the search. It has been shown that most people do not look beyond the first three pages of search results $[5,19]$. While YouTube used to post 20 videos on each page, it now presents search results in a continuous list. Therefore, we sampled the top 60 videos for each search term. All selected videos were added to the YouTube library database for further analysis.

Only videos presented in the English language were included in the study considering that it is assumed to be a universal language by many countries worldwide [18]. Duplicate videos, irrelevant videos, music videos, and videos without audio were excluded. Of the total 240 videos screened, 114 met the inclusion criteria and analyzed blindly by two rheumatologists (M.E.O. and O.Z.). Any discrepancy between the authors was resolved by reassessment and consensus. The title of the video, upload date, duration, and number of views, views per day, likes, dislikes and comments were extracted by the authors. The video sources were classified into five categories as academic institutions/ professional organizations, physicians, healthcare professionals other than physicians (e.g., dietitians, nurses, and physical therapists), health information websites, and independent users. The videos were also grouped according to the target audience as patients, healthcare professionals, and unspecified.

\section{Evaluation of usefulness}

The videos meeting the inclusion criteria were evaluated in terms of usefulness and categorized as useful and misleading. In accordance with previous studies [7, 20, 21], the videos were considered as useful if they included scientifically accurate information about any aspect of the disease (e.g., symptoms, clinical features, diagnosis, treatment, and prevention) or misleading if they included at least one scientifically unproven information.

\section{Evaluation of reliability and quality}

Video reliability was evaluated using a modified five-point DISCERN tool, which has been previously used in several studies $[8,9,22]$. This questionnaire was adapted by Singh et al. from the original DISCERN tool [5]. Each question is answered as 'yes' or 'no', scored as 1 point and 0 point, respectively. Higher scores represent greater reliability.

Overall video quality was assessed using the five-point Global Quality Scale (GQS), which was also used in numerous YouTube studies previously [1, 8, 9]. Higher scores indicate better video quality.

\section{Ethical statement}

Publicly available YouTube videos were analyzed, and no human participants or animals were recruited for the study; therefore, ethical approval was not required, similar to other similar YouTube studies [22-24].

\section{Statistical analysis}

The Shapiro-Wilk test was performed to assess the normality of data. The inter-observer agreement was assessed with Cohen's kappa coefficient. Non-normally distributed continuous variables were expressed as median (minimum-maximum) values. Categorical variables were presented as number or percentages. Pairwise comparisons were performed between the 'useful' and 'misleading' videos using the Mann-Whitney $U$ test. The reliability and quality scores according to video sources were compared using the Kruskal-Wallis test. In cases where a significant difference was found in the Kruskal-Wallis test, pairwise comparisons were evaluated using the Mann-Whitney $U$ test with Bonferroni correction that automatically adjusted by SPSS version 22. A $p$ value of less than 0.05 was considered as statistically significant. Statistical tests were 
performed using SPSS v. 22.0 for Windows (IBM SPSS Statistics for Windows; Armonk, NY: IBM Corp).

\section{Results}

Of the total 240 videos screened, 95 duplicates, 13 videos presented in a language other than English, nine irrelevant videos, eight music videos, and one video with no audio were excluded from the study, and as a result 114 videos were identified for further analysis (Fig. 1). Cohen's kappa statistic demonstrating inter-observer agreement was 0.925 [95\% confidence interval (CI): 0.883-1.000]. The median duration of the videos was 4.31 (range, 0.31-97.15) minutes. The median number of views was $17,353.50$ (range $40-3,114,612$ ) and the median number of views per day was 24.86 (0.09-2974). The median number of days since upload was 916.50 (range 28-4429). The baseline features of the videos are encapsulated in Table 1 .

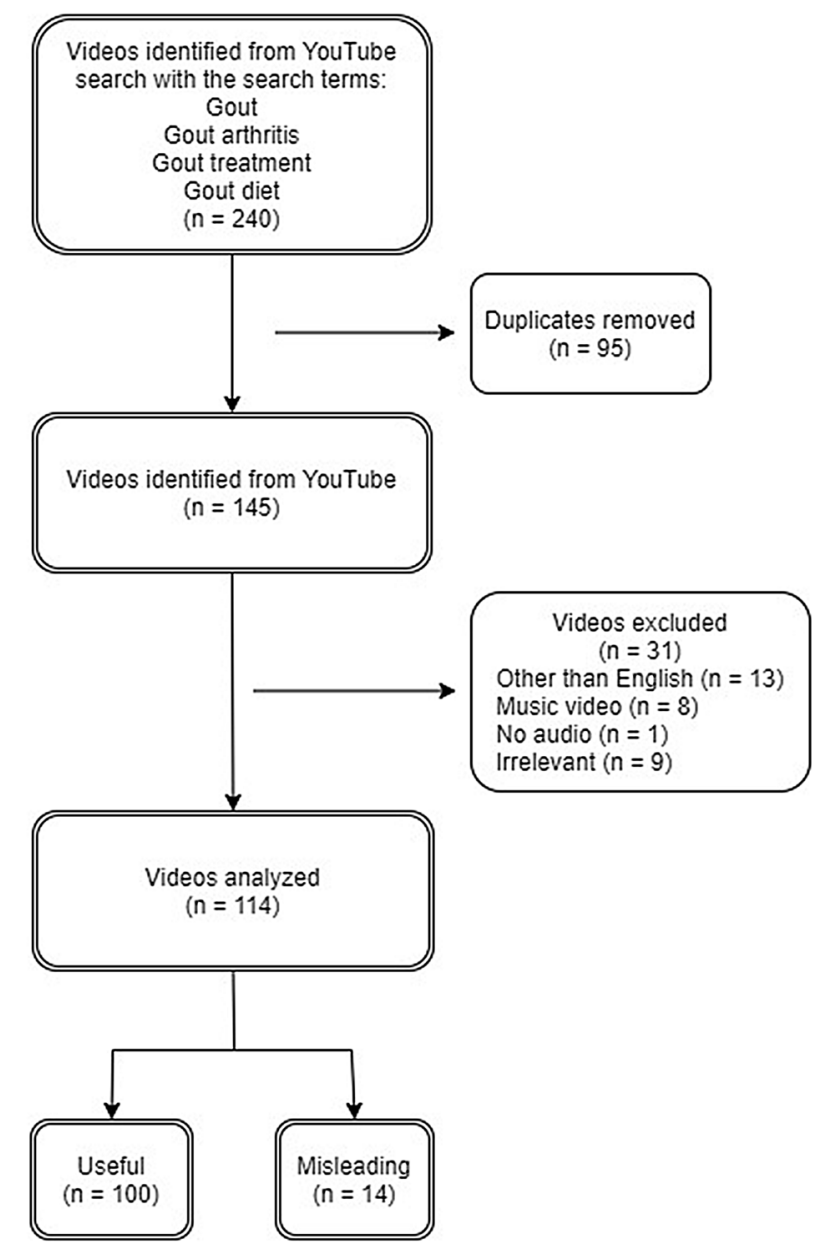

Fig. 1 Flowchart showing the selection of YouTube videos on gout
Table 1 Baseline features of the analyzed videos

\begin{tabular}{ll}
\hline Variables & Videos $(n=114)$ \\
\hline Number of days on YouTube & $916.50(28-4429)$ \\
Number of views & $17,353.50(40-3,114,612)$ \\
Number of views per day & $24.86(0.09-2974)$ \\
Number of likes & $183.00(0-26,000)$ \\
Number of dislikes & $12.00(0-1900)$ \\
Number of comments & $23(0-1205)$ \\
Duration (minute) & $4.31(0.31-97.15)$ \\
DISCERN & $3.00(1-5)$ \\
GQS & $4.00(1-5)$ \\
\hline
\end{tabular}

Data presented as median (minimum-maximum) values

DISCERN modified DISCERN score, GQS Global Quality Scale score

\section{Video content and presenters}

Of the total 114 videos, 32 (28.07\%) contained information on "gout diet", followed by 24 (21.05\%) on "gout treatment," and $24(21.05 \%)$ on "acute gout attack and gout arthritis". Other videos comprised an overview of gout (14.04\%), its pathophysiology (4.39\%), associated comorbidities (7.02\%), and gout surgery $(4.39 \%)$. The content of the analyzed videos is demonstrated in Table 2.

Among the 114 videos analyzed, the majority of the main presenters were rheumatologists $(n=26,22.8 \%)$, followed by orthopedists $(n=15,10.5 \%)$ and podiatrists $(n=9,7.9 \%)$. Other physicians who contributed to the videos included family medicine physicians, general practitioners, biochemists, pharmacologists, internists, immunologists, physiatrists, and sports medicine physicians. Among non-physician healthcare professionals were dieticians, nutritionists, nurses, and physical therapists.

\section{Video usefulness}

Of the analyzed videos, $87.72 \%$ (100/114) provided useful and $12.28 \%(14 / 114)$ contained misleading information.

Table 2 Video contents

\begin{tabular}{lc}
\hline 1. An overview of gout & $16(14.04 \%)$ \\
2. Pathophysiology of gout & $5(4.39 \%)$ \\
3. Acute gout attack and gout arthritis & $24(21.05 \%)$ \\
4. Gout treatment & $24(21.05 \%)$ \\
5. Gout diet & $32(28.07 \%)$ \\
6. Associated comorbidities & $8(7.02 \%)$ \\
7. Gout surgery-removal of gout crystals & $5(4.39 \%)$ \\
\hline
\end{tabular}

Categorical variables given as numbers ( $n$ ) and percentages (\%) More than one topic may be mentioned in a single video 
Independent user-created videos displayed the highest percentage of misleading information at $57.14 \%$ (4/7), whereas all the videos created by the academic institutions/professional organizations were useful. There was no significant difference in the mean number of likes and comments and video length between the useful and misleading videos. The mean number of views and number of views per day were significantly higher in misleading videos than useful ones ( $p=0.032$ and $p=0.049$, respectively). The mean number of dislikes was significantly higher in misleading videos $(p=0.038)$. The useful videos had higher scores for reliability and quality obtained by modified DISCERN (3.0, range $1.0-5.0$ vs 2.0 , range $1.0-4.0)$ and GQS (4.0, range $1.0-5.0$ vs 2.0 , range 1.0-4.0) compared to the misleading videos, and this was statistically significant $(p<0.001$ for both). The detailed characteristics of the YouTube videos according to their usefulness are presented in Table 3.

\section{Targeted audience and video source}

The most targeted audience of all videos was patients $(n=81,71.05 \%)$, followed by healthcare professionals
( $n=26,22.81)$. Although the percentage of useful videos targeting patients was $83.95 \%$, this rate was higher for videos targeting healthcare professionals $(96.15 \%)$. The majority of the videos on YouTube had been posted by physicians $(n=42,36.84 \%)$, followed by health information websites $(n=35,30.70 \%)$, academic institutions/professional organizations ( $n=20,17.54 \%)$, healthcare professionals other than physicians $(n=10,8.77 \%)$, and independent users $(n=7$, (6.14\%), respectively.

\section{Reliability and quality of the videos}

The videos uploaded by academic institutions/professional organizations had higher modified DISCERN and GQS scores than those from all the remaining video sources, and the difference was statistically significant $(p<0.001$ for both), except in the comparison between academic institutions/professional organizations and physicians. The mean modified DISCERN scores posted by academic institutions/professional organizations were significantly higher than those provided by healthcare professionals other than physicians, health information websites, and independent

Table 3 Detailed characteristics of YouTube videos according to their usefulness

\begin{tabular}{|c|c|c|c|}
\hline & $\begin{array}{l}\text { Useful } \\
100(87.72 \%)\end{array}$ & $\begin{array}{l}\text { Misleading } \\
14(12.28 \%)\end{array}$ & $p$ \\
\hline \multicolumn{4}{|l|}{ Variables } \\
\hline Number of views & $15,555(40-3,114,612)$ & $67,134(4689-180,654)$ & $0.032 *$ \\
\hline Number of views per day & $23.43(0.09-2974)$ & $41.40(9.82-241.48)$ & $0.049 *$ \\
\hline Number of likes & $148(0-26,000)$ & $328(37-2400)$ & 0.161 \\
\hline Number of dislikes & $11(0-1900)$ & $33.5(2-341)$ & 0.038* \\
\hline Number of comments & $18.5(0-1205)$ & $68.5(4-574)$ & 0.057 \\
\hline Duration (minutes) & $4.34(0.31-97.15)$ & $3.09(1.3-13.15)$ & 0.163 \\
\hline \multicolumn{4}{|l|}{ Reliability and quality scores } \\
\hline \multicolumn{4}{|l|}{ Median $(\min -\max )$} \\
\hline DISCERN & $3.0(1.0-5.0)$ & $2.0(1.0-4.0)$ & $<0.001 *$ \\
\hline GQS & $4.0(1.0-5.0)$ & $2.0(1.0-4.0)$ & $<0.001 *$ \\
\hline \multicolumn{4}{|l|}{ Target audience } \\
\hline Patients & $68(83.95 \%)$ & $13(16.05 \%)$ & N/A \\
\hline Healthcare professionals & $25(96.15 \%)$ & $1(3.85 \%)$ & N/A \\
\hline Unspecified & $7(100 \%)$ & $0(0 \%)$ & N/A \\
\hline \multicolumn{4}{|l|}{ Source, $n(\%)$} \\
\hline Academic institutions/professional organizations & $20(100 \%)$ & $0(0 \%)$ & N/A \\
\hline Physicians & $40(95.24 \%)$ & $2(4.76 \%)$ & N/A \\
\hline Health information websites & $30(85.71 \%)$ & $5(14.29 \%)$ & N/A \\
\hline Healthcare professionals other than physicians & $7(70 \%)$ & $3(30 \%)$ & N/A \\
\hline Independent users & $3(42.86 \%)$ & $4(57.14 \%)$ & N/A \\
\hline
\end{tabular}

Data presented as $n$ (\%) or median (minimum-maximum)

Comparisons between the 'useful' and 'misleading' videos were performed using the Mann-Whitney $U$ test

DISCERN modified DISCERN score, GQS Global Quality Scale score, N/A not applicable

*Values of $p<0.05$ were accepted as significant and marked bold 
users ( $p<0.001, p=0.031$, and $p=0.001$, respectively). The mean GQS scores of the videos created by academic institutions/professional organizations were significantly higher than those posted by healthcare professionals other than physicians, health information websites, and independent users ( $p<0.001, p=0.004$, and $p<0.001$, respectively). The comparison of the reliability and quality scores according to video sources is presented in Table 4.

GQS was evaluated in three categories, with scores of 1-2 indicating low quality, 3 intermediate quality and 4-5 high quality [22]. The majority of the videos demonstrated high quality $(57.89 \%)$, followed by intermediate quality $(28.95 \%)$, while the percentage of low-quality videos was $13.16 \%$ (Fig. 2).

\section{Discussion}

Today, patients increasingly turn to online sources to make informed decisions [1]. The internet is the third most trustworthy source of health information after physicians and government healthcare institutions and is perceived as more reliable than traditional mass media [25]. Patient education is often emphasized as a vital determinant in order to optimize outcomes for chronic rheumatic diseases, such as gout [9]. YouTube is a free and easy-to-access video sharing platform with the potential of being a valuable source of healthrelated information [1]. However, the variety of origin and lack of a filter process on this platform can lead to the spread of misleading information [1]. The aim of this study was to evaluate the reliability and quality of gout-related videos on YouTube.

We analyzed 114 videos with a total view count of over 16 million and a whole duration of $19.3 \mathrm{~h}$, indicating YouTube as a popular source of information for gout patients. The earliest video in the sample was posted on October 21, 2010, and patients were the primary target audience (71\%). Rheumatologists were the most common specialists in the analyzed videos. Hay et al. found that $87.5 \%$ of rheumatology patients referred to the internet to seek information about their condition before their first appointment with a rheumatologist [26]. In addition, the authors found that

\section{Video Quality About Gout on YouTube}

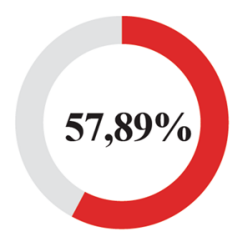

\section{High Quality}

More than half of the videos provided high quality.

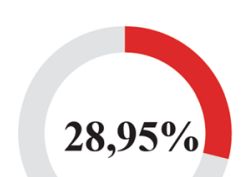

\section{Intermediate Quality}

Nearly one-third of the videos provided moderate quality deemed as acceptable.

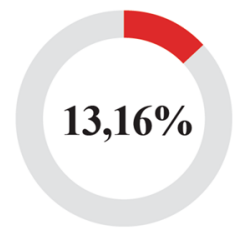

\section{Low Quality}

The proportion of videos with poor quality was low.

Fig. 2 Quality of the analyzed videos according to Global Quality Scale

patients with more pain tended to resort to the internet more to obtain information [26]. Considering that gout patients experience severe pain, it is not unexpected for these patients to seek online information quite often. The major advantage of YouTube compared to a face-to-face meeting with a physician is that it can be easily accessed with a click for $24 \mathrm{~h}$ a day, seven days a week. Consequently, YouTube can be a source of information to complement the physician's advice, given the limited time for consultation.

When we analyzed the content of the videos, we determined that the most frequently addressed topic was an appropriate diet for gout (28.07\%). The following topics were gout arthritis and gout treatment (21.05\% for both). Although many physicians have focused on urate-lowering

Table 4 Comparison of the reliability and quality scores according to video sources

\begin{tabular}{lllllll}
\hline $\begin{array}{l}\text { Reliability and } \\
\text { quality scores }\end{array}$ & $\begin{array}{l}\text { Academic institutions/pro- } \\
\text { fessional organizations } \\
20(17.54 \%)\end{array}$ & Physicians & $\begin{array}{l}\text { Healthcare professionals } \\
\text { other than physicians } \\
10(8.77 \%)\end{array}$ & $\begin{array}{l}\text { Health informa- } \\
\text { tion websites }\end{array}$ & $\begin{array}{l}\text { Independent users } \\
35(30.70 \%)\end{array}$ & $7(6.14 \%)$ \\
\hline DISCERN & $5.0(5.0-5.0)$ & $4.0(1.0-5.0)$ & $3.0(1.0-4.0)$ & $3.0(1.0-5.0)$ & $2.0(1.0-2.0)$ \\
GQS & $5.0(4.0-5.0)$ & $4.0(2.0-5.0)$ & $3.0(1.0-4.0)$ & $3.0(1.0-4.0)$ & $2.0(1.0-2.0)$ & $<\mathbf{0 . 0 0 1 *}$ \\
\hline
\end{tabular}

DISCERN modified DISCERN score, GQS Global Quality Scale score

${ }^{*} p<0.05$ was accepted as significant (Kruskal-Wallis test) and marked bold 
therapy, diet remains a very crucial part of gout management [27], which is also emphasized by the guidelines of professional organizations such as ACR and EULAR $[15,16]$. It has been supported by strong evidence that the main component of lifestyle changes required in gout is weight loss through diet modification [27]. The absence of symptoms between arthritis attacks may make patients feel complacent about their condition and lead them to seek improvement with diet rather than medication. Therefore, it is not unexpected to determine diet as the most common topic. However, an interesting finding was that content related to associated comorbidities had a relatively small proportion (7.02\%). Given its importance in gout management, this content should be further addressed, especially by academic institutions/professional organizations.

Video engagement parameters such as the number of likes and comments did not statistically significantly differ between the useful and misleading videos, which is consistent with previous studies on Sjogren's syndrome, rheumatoid arthritis, spondyloarthritis, and systemic lupus erythematosus [1, 5, 8, 9]. However, previous studies displayed variations in viewership between useful and misleading videos. Elangovan et al. [8] and Esen et al. [28] observed no significant differences in the viewership between useful and misleading videos. In contrast, Garg et al. [29] demonstrated higher viewership for misleading videos similar to our study. Thus, more view count does not always mean accurate information. Interestingly, we found that the number of dislikes was statistically significant in the misleading videos than the useful ones, suggesting that patients may have become aware of the misleading nature of the videos after watching them. To eliminate this issue, YouTube should consider establishing a prevention mechanism before uploading videos.

A recent study about information and misinformation on COVID-19 revealed that social media channels were the most important source of misinformation [30]. While social media platforms are easily accessible sources of healthrelated information, they do not always provide appropriate content. YouTube, a popular social media platform, contains misleading information that contradicts the guidelines and standards in the field, and non-healthcare users are more vulnerable to such content [2]. While $87.72 \%$ of the videos analyzed in our study included accurate health information and were deemed as useful, $12.28 \%$ contained misleading information. This percentage of useful information was higher than those reported by previous studies (58.3-63\%) assessing medical health information on YouTube videos related to hypertension, dialysis, and influenza [29, 31, 32]. In studies that recently analyzed misinformation about COVID-19 on YouTube [7, 18, 33], the percentage of useful videos was also determined to be lower (37.14-72.50\%) compared to our study. Concerning rheumatic diseases, YouTube studies have shown varying results; for example, the rate of useful videos was reported as $51.4 \%$ for Sjogren's syndrome [1] and 54.9\% for rheumatoid arthritis [5], which were both lower than our study, while studies on spondyloarthritis and systemic lupus erythematosus determined similar rates to our study ( $86 \%$ and $83.60 \%$, respectively) $[8,9]$. In our study, no misleading videos were posted by academic institutions/professional organizations, which is in agreement with previous studies [7, 18, 21, 33]. Not surprisingly, the modified DISCERN and GQS scores for the useful videos were significantly higher than those of the misleading videos $(p<0.001)$. Most of the videos in our study contained useful information with acceptable reliability and quality, suggesting that gout information presented on YouTube can be beneficial for both patients and healthcare professionals. However, specialists interested in gout should be aware that YouTube may contain some misinformation, and patients may be exposed to unscientific content.

According to sources, the majority of the videos had been uploaded by physicians (36.84\%) while independent users had posted only a smaller portion of the videos $(6.14 \%)$. Academic institutions/professional organizations had uploaded $17.54 \%$ of the total videos, which is slightly higher than reported in previous studies $(10-15 \%)[5,18,29]$. In the literature, the highest scores for modified DISCERN and GQS were considered to indicate the highest reliability and quality [1,9], which was also the case in the current study. In addition, the reliability and quality scores of the videos uploaded by academic institutions/professional organizations were significantly higher compared to the other video sources except physicians. The median modified DISCERN and GQS scores of the videos provided by healthcare professionals other than physicians and health information websites were both 3 , indicating moderate reliability and quality. The videos posted by independent users demonstrated the lowest reliability and quality scores, as expected based on previous studies [18, 23, 34]. A study by Rice demonstrated that $25 \%$ of people seeking health information always checked the video source, $25 \%$ checked it sometimes, and $50 \%$ never checked it [35]. Physicians should consider advising their patients to view the source when searching the internet to increase the possibility of accessing reliable and high-qualify medical information on gout.

\section{Limitations}

This was a cross-sectional study that captured only an instantaneous snapshot. YouTube is a dynamic video-sharing platform with daily renewed content, and therefore this limitation is expected for all similar studies. Another limitation was that we analyzed only English-language videos. However, English is accepted as the prevailing language for access to online information [1]. Our evaluation was also limited to the first 60 videos for each search term. We 
decided on this number based on previous data suggesting that the majority of consumers do not look beyond the first few pages when searching YouTube [36]. Lastly, only YouTube videos were analyzed, and other social media websites were not evaluated.

\section{Conclusion}

Our study revealed that the majority of YouTube videos on gout provided useful information. This information may help increase patients' awareness about gout arthritis, treatment decisions, and diet, and support the information provided by physicians. However, physicians should be aware of the limitations of YouTube videos to better guide their patients, and they should also correct any misinformation during face-to-face meetings with their patients. Professional organizations such as EULAR and ACR should consider collaborating with YouTube to increase the dissemination of accurate, evidence-based information for rheumatic diseases. YouTube should consider blocking misleading videos using validity scales such as modified DISCERN and GQS.

Author contributions The authors contributed equally to researching data for the article, discussion of its content, writing, reviewing and editing of the manuscript before submission.

Funding Author Mustafa Erkut Onder and author Orhan Zengin declare that there has been no financial support for this work.

Availability of data and material The authors confirm that the data supporting the findings of this study are available within the article and its supplementary materials.

\section{Declarations}

Conflict of interest The authors declare that they have no conflict of interest.

Ethics approval Publicly accessible YouTube videos were used and no human participants were involved in the study; hence, ethical approval was not required.

\section{References}

1. Delli K, Livas C, Vissink A, Spijkervet FK (2016) Is YouTube useful as a source of information for Sjögren's syndrome? Oral Dis 22(3):196-201. https://doi.org/10.1111/odi.12404

2. Madathil KC, Rivera-Rodriguez AJ, Greenstein JS, Gramopadhye AK (2015) Healthcare information on YouTube: a systematic review. Health Inform J 21(3):173-194. https://doi. org/10.1177/1460458213512220

3. Wikipedia The Free Encyclopedia. List of most popular websites. https://en.wikipedia.org/wiki/List_of_most_popular_websites. Accessed 11 Dec 2020
4. YouTube for press. https://blog.youtube/press/. Accessed 12 Dec 2020

5. Singh AG, Singh S, Singh PP (2012) YouTube for information on rheumatoid arthritis-a wakeup call? J Rheumatol 39(5):899_ 903. https://doi.org/10.3899/jrheum.111114

6. Koller U, Waldstein W, Schatz KD, Windhager R (2016) YouTube provides irrelevant information for the diagnosis and treatment of hip arthritis. Int Orthop 40(10):1995-2002. https://doi. org/10.1007/s00264-016-3174-7

7. Moon H, Lee GH (2020) Evaluation of Korean-language COVID-19-related medical information on YouTube: CROSSSECTIONAL INFODEMIOLOGY STUDy. J Med Internet Res 22(8):e20775. https://doi.org/10.2196/20775

8. Elangovan S, Kwan YH, Fong W (2020) The usefulness and validity of English-language videos on YouTube as an educational resource for spondyloarthritis. Clin Rheumatol. https:// doi.org/10.1007/s10067-020-05377-w

9. Ng CH, Lim GRS, Fong W (2020) Quality of English-language videos on YouTube as a source of information on systemic lupus erythematosus. Int J Rheum Dis 23(12):1636-1644. https://doi. org/10.1111/1756-185X.13852

10. Richette P, Bardin T (2010) Gout. Lancet 375(9711):318-328. https://doi.org/10.1016/S0140-6736(09)60883-7

11. Dalbeth N, Choi HK, Joosten LAB, Khanna PP, Matsuo H, Perez-Ruiz F, Stamp LK (2019) Gout. Nat Rev Dis Primers 5(1):69. https://doi.org/10.1038/s41572-019-0115-y

12. Dalbeth N, Merriman TR, Stamp LK (2016) Gout. Lancet 388(10055):2039-2052. https://doi.org/10.1016/S0140 $-6736(16) 00346-9$

13. Tang SCW (2018) Gout: a disease of kings. Contrib Nephrol 192:77-81. https://doi.org/10.1159/000484281

14. Abhishek A, Doherty M (2018) Education and non-pharmacological approaches for gout. Rheumatology (Oxford) 57(suppl_1):i51-i58. https://doi.org/10.1093/rheumatology/ kex421

15. FitzGerald JD, Dalbeth N, Mikuls T, Brignardello-Petersen R, Guyatt G, Abeles AM, Gelber AC, Harrold LR, Khanna D, King C, Levy G, Libbey C, Mount D, Pillinger MH, Rosenthal A, Singh JA, Sims JE, Smith BJ, Wenger NS, Bae SS, Danve A, Khanna PP, Kim SC, Lenert A, Poon S, Qasim A, Sehra ST, Sharma TSK, Toprover M, Turgunbaev M, Zeng L, Zhang MA, Turner AS, Neogi T (2020) 2020 American College of Rheumatology Guideline for the Management of Gout. Arthritis Care Res (Hoboken) 72(6):744-760. https://doi.org/10.1002/acr.24180

16. Richette P, Doherty M, Pascual E, Barskova V, Becce F, Castañeda-Sanabria J, Coyfish M, Guillo S, Jansen TL, Janssens H, Lioté F, Mallen C, Nuki G, Perez-Ruiz F, Pimentao J, Punzi L, Pywell T, So A, Tausche AK, Uhlig T, Zavada J, Zhang W, Tubach F, Bardin T (2017) 2016 updated EULAR evidence-based recommendations for the management of gout. Ann Rheum Dis 76(1):29-42. https://doi.org/10.1136/annrheumdis-2016-209707

17. Robinson PC, Stamp LK (2016) The management of gout: Much has changed. Aust Fam Physician 45(5):299-302

18. Li HO, Bailey A, Huynh D, Chan J (2020) YouTube as a source of information on COVID-19: a pandemic of misinformation? BMJ Glob Health 5(5):e002604. https://doi.org/10.1136/bmjgh $-2020-002604$

19. Dutta A, Beriwal N, Van Breugel LM, Sachdeva S, Barman B, Saikia H, Nelson UA, Mahdy A, Paul S (2020) YouTube as a source of medical and epidemiological information during COVID-19 pandemic: a cross-sectional study of content across six languages around the globe. Cureus 12(6):e8622. https://doi. org/10.7759/cureus.8622

20. Khatri P, Singh SR, Belani NK, Yeong YL, Lohan R, Lim YW, Teo WZ (2020) YouTube as source of information on 2019 novel coronavirus outbreak: a cross sectional study of English and 
Mandarin content. Travel Med Infect Dis 35:101636. https://doi. org/10.1016/j.tmaid.2020.101636

21. Pathak R, Poudel DR, Karmacharya P, Pathak A, Aryal MR, Mahmood M, Donato AA (2015) YouTube as a source of information on Ebola Virus Disease. N Am J Med Sci 7(7):306-309. https://doi.org/10.4103/1947-2714.161244

22. Kocyigit BF, Akaltun MS, Sahin AR (2020) YouTube as a source of information on COVID-19 and rheumatic disease link. Clin Rheumatol 39(7):2049-2054. https://doi.org/10.1007/s1006 7-020-05176-3

23. Hernández-García I, Giménez-Júlvez T (2020) Characteristics of YouTube Videos in Spanish on How to Prevent COVID-19. Int J Environ Res Public Health 17(13):4671. https://doi.org/10.3390/ ijerph17134671

24. Nason GJ, Kelly P, Kelly ME, Burke MJ, Aslam A, Giri SK, Flood HD (2015) YouTube as an educational tool regarding male urethral catheterization. Scand J Urol 49(2):189-192. https://doi. org $/ 10.3109 / 21681805.2014 .975837$

25. Ye Y (2010) A path analysis on correlates of consumer trust in online health information: evidence from the health information national trends survey. J Health Commun 15(Suppl 3):200-215. https://doi.org/10.1080/10810730.2010.522687

26. Hay MC, Cadigan RJ, Khanna D, Strathmann C, Lieber E, Altman R, McMahon M, Kokhab M, Furst DE (2008) Prepared patients: internet information seeking by new rheumatology patients. Arthritis Rheum 59(4):575-582. https://doi.org/10.1002/art.23533

27. Beyl RN Jr, Hughes L, Morgan S (2016) Update on importance of diet in gout. Am J Med 129(11):1153-1158. https://doi. org/10.1016/j.amjmed.2016.06.040

28. Esen E, Aslan M, Sonbahar BÇ, Kerimoğlu RS (2019) YouTube English videos as a source of information on breast self-examination. Breast Cancer Res Treat 173(3):629-635. https://doi. org/10.1007/s10549-018-5044-Z

29. Garg N, Venkatraman A, Pandey A, Kumar N (2015) YouTube as a source of information on dialysis: a content analysis. Nephrology (Carlton) 20(5):315-320. https://doi.org/10.1111/nep.12397
30. Gupta L, Gasparyan AY, Misra DP, Agarwal V, Zimba O, Yessirkepov M (2020) Information and misinformation on COVID19: a cross-sectional survey study. J Korean Med Sci 35(27):e256. https://doi.org/10.3346/jkms.2020.35.e256

31. Pandey A, Patni N, Singh M, Sood A, Singh G (2010) YouTube as a source of information on the H1N1 influenza pandemic. Am J Prev Med 38(3):e1-3. https://doi.org/10.1016/j.amepr e.2009.11.007

32. Kumar N, Pandey A, Venkatraman A, Garg N (2014) Are video sharing web sites a useful source of information on hypertension? J Am Soc Hypertens 8(7):481-490. https://doi.org/10.1016/j. jash.2014.05.001

33. D'Souza RS, D'Souza S, Strand N, Anderson A, Vogt MNP, Olatoye O (2020) YouTube as a source of medical information on the novel coronavirus 2019 disease (COVID-19) pandemic. Glob Public Health 15(7):935-942. https://doi.org/10.1080/17441 692.2020.1761426

34. Kocyigit BF, Nacitarhan V, Koca TT, Berk E (2019) YouTube as a source of patient information for ankylosing spondylitis exercises. Clin Rheumatol 38(6):1747-1751. https://doi.org/10.1007/s1006 7-018-04413-0

35. Rice RE (2006) Influences, usage, and outcomes of Internet health information searching: multivariate results from the Pew surveys. Int J Med Inform 75(1):8-28. https://doi.org/10.1016/j.ijmed inf.2005.07.032

36. Villafañe JH, Cantero-Tellez R, Valdes K, Usuelli FG, Berjano P (2018) Educational quality of YouTube videos in thumb exercises for carpometacarpal osteoarthritis: a search on current practice. Hand (N Y) 13(6):715-719. https://doi.org/10.1177/1558944717 726139

Publisher's Note Springer Nature remains neutral with regard to jurisdictional claims in published maps and institutional affiliations. 